\title{
Deletion of the EphA2 receptor exacerbates myocardial injury and the progression of ischemic cardiomyopathy
}

\author{
Wesley T. O’Neal', William F. Griffin², Susan D. Kent ${ }^{2}$, Filza Faiz², Jonathan Hodges², \\ Jackson Vuncannon ${ }^{2}$ and Jitka A. I. Virag ${ }^{2 *}$
}

Department of Internal Medicine, Wake Forest School of Medicine, Winston-Salem, NC, USA

${ }^{2}$ Department of Physiology, Brody School of Medicine, East Carolina University, Greenville, NC, USA

\section{Edited by:}

Ovidiu Constantin Baltatu, University

Camilo Castelo Branco, Brazil

\section{Reviewed by:}

Thomas Langenickel, Novartis Institutes for Biomedical Research, Switzerland

Luciana Lopes Guimaraes,

Universidade Santa Cecilia, Brazil

\section{${ }^{*}$ Correspondence:}

Jitka A. I. Virag, Department of

Physiology, LSB-239, Brody School

of Medicine, East Carolina

University, 600 Moye Blvd.,

Greenville, NC 27834, USA

e-mail:viragj@ecu.edu
EphrinA1-EphA-receptor signaling is protective during myocardial infarction (MI). The EphA2-receptor (EphA2-R) potentially mediates cardiomyocyte survival. To determine the role of the EphA2-R in acute non-reperfused myocardial injury in vivo, infarct size, inflammatory cell density, NF-kB, p-AKT/Akt, and MMP-2 protein levels, and changes in ephrinA1/EphA2-R gene expression profile were assessed 4 days post-MI in B6129 wild-type (WT) and EphA2-R-mutant (EphA2-R-M) mice lacking a functional EphA2-R. Fibrosis, capillary density, morphometry of left ventricular chamber and infarct dimensions, and cardiac function also were measured 4 weeks post-Ml to determine the extent of ventricular remodeling. EphA2-R-M infarct size and area of residual necrosis were 31.7\% and $113 \%$ greater than WT hearts, respectively. Neutrophil and macrophage infiltration were increased by $46 \%$ and $84 \%$ in EphA2-R-M hearts compared with WT, respectively. NF-kB protein expression was 1.9-fold greater in EphA2-R-M hearts at baseline and 56\% less NF-kB after infarction compared with WT. EphA6 gene expression was 2.5-fold higher at baseline and increased 9.8-fold 4 days post-MI in EphA2-R-M hearts compared with WT. EphrinA1 gene expression in EphA2-R-M hearts was unchanged at baseline and decreased by $42 \% 4$ days post-MI compared with WT hearts. EphA2-R-M hearts had $66.7 \%$ less expression of total Akt protein and 59\% less p-Akt protein than WT hearts post-MI. EphA2-R-M hearts 4 weeks post-MI had increased chamber dilation and interstitial fibrosis and decreased MMP-2 expression and capillary density compared with WT. In conclusion, the EphA2-R is necessary to appropriately modulate the inflammatory response and severity of early injury during acute $\mathrm{Ml}$, thereby influencing the progression of ischemic cardiomyopathy.

Keywords: myocardial infarction, EphA2, inflammation, remodeling, ischemia

\section{INTRODUCTION}

Myocardial Infarction (MI) is associated with an increased risk of congestive heart failure. After the initial infarction, structural remodeling ensues, leading to progressive ventricular dilation and dysfunction (Kannel, 2000; Frangogiannis et al., 2002; Jugdutt, 2012). Manipulation of cell signaling represents a new therapeutic approach to combat the development of heart failure by reducing the severity of the original ischemic insult (French et al., 2010; Kung et al., 2011).

The Eph receptors (Eph-R) and their ligands, the ephrins, are the largest family of receptor tyrosine kinases. Ephrin-Eph-R signaling regulates cell differentiation, proliferation, and migration during development (Kullander and Klein, 2002; Pasquale, 2008) and plays a pivotal role in various cancers (Brantley et al., 2002; McCarron et al., 2010). EphrinAl, an angiogenic protein stimulated by pro-inflammatory cytokines, promotes tumorigenesis through interaction with the EphA2-R (Pandey et al., 1995; Cheng et al., 2002; Brantley-Sieders et al., 2004a,b, 2006; Pasquale, 2008; Miao et al., 2009; Beauchamp and Debinski, 2011). Antagonism of the EphA2-R with lithocholic acid blocks EphA2-R phosphorylation and reduces cancer cell growth (Incerti et al., 2013).
Furthermore, the absence of the EphA2-R in mice confers a predilection for hyper-responsiveness to allergens demonstrated by increased inflammation (Okazaki et al., 2009). Conversely, EphA2-R in other tissues has been demonstrated to enhance inflammation via endothelial cell priming and inflammatory cell migration (Ivanov and Romanovsky, 2006; Coulthard et al., 2012; Funk et al., 2012; Funk and Orr, 2013). EphA2-R expression on human monocytes and coronary artery endothelial cells, both of which express a combination of other ephrinA ligands and EphA receptors, may modulate inflammatory cell transmigration (Sakamoto et al., 2011). Also, EphA2-R is expressed on rat vascular smooth muscle cells and contributes to ephrinA1-mediated vessel destabilization during revascularization (Deroanne et al., 2003). The tissue- and cell-specific role of EphA2-R and its interaction with other EphA-Rs and ephrinA ligands in myocardial injury is complex and warrants investigation.

Recently, our lab and others have reported that ephrinA1EphA-R signaling can be manipulated to preserve cardiomyocyte function after MI. We have shown that ephrinA1-Fc administration is cardioprotective during $\mathrm{MI}$ and possibly this, at 
least in part, is mediated through the EphA2-R (Dries et al., 2011). Goichberg et al. has shown that ephrinA1-Fc treatment during MI is cardioprotective through EphA2-R-mediated revascularization and regeneration (Goichberg et al., 2011). Jehle et al. demonstrated that immortalized HL-1 cardiomyocytes exposed to doxazocin were protected from apoptosis by increased EphA2-R expression and reduced phosphorylation in response to lithocholic acid (Jehle et al., 2012). Combined with its known pro-angiogenic and pro-inflammatory actions in the heart, we hypothesized that MI in mice lacking a functional EphA2-R worsens cardiomyocyte injury and inflammation leading to adverse remodeling and cardiac dysfunction (O'Neal et al., 2013). To investigate this, we examined the acute response to non-reperfused MI 4 days post-MI and the progression of ischemic cardiomyopathy 4 weeks post-MI in EphA2-R-mutant (EphA2-R-M) mice.

\section{METHODS}

\section{ANIMALS}

Experimental research protocols were approved by the East Carolina University Institutional Animal Care and Use Committee following the guidelines of the National Institutes of Health for the Care and Use of Laboratory Animals. B6129SF2/J mice (stock \#101045) (WT) and B6129S6-Epha2 ${ }^{\text {tm1Jrui } / J ~(s t o c k ~}$ \#006028; an EphA2-deficient homozygous mutant (truncated, non-functional protein) (Brantley-Sieders et al., 2004a,b); EphA2-R-M) mice were purchased from Jackson Laboratories. All mice were housed in ventilated cages and animal care was maintained by the Department of Comparative Medicine at The Brody School of Medicine, East Carolina University. Mice were exposed to 12/12 hour light/dark cycle conditions and received food and water ad-libitum.

\section{SURGICAL PROCEDURE AND TISSUE COLLECTION}

Male WT and EphA2-R-M mice (8-12 weeks) were anesthetized with an intraperitoneal injection of $20 \mu \mathrm{l} / \mathrm{g}$ body weight Avertin $(20 \mathrm{mg} / \mathrm{ml})$ and mechanically ventilated. The left anterior descending coronary artery was permanently occluded using an $8-0$ suture. The rib cage, muscle, and skin were then closed with a 6-0 suture. Four days or 4 weeks after surgery, mice were anesthetized with a lethal intraperitoneal injection of $0.1 \mathrm{~mL}$ pentobarbital $(390 \mathrm{mg} / \mathrm{mL})$. At the time of sacrifice, the heart was arrested in diastole using cold $\mathrm{KCl}(30 \mathrm{mM})$, excised, rinsed in saline, and immersed in zinc fixative or snap frozen in liquid nitrogen. For immunohistochemical staining and morphometric measurements, whole hearts were transversely sectioned into 4 slices of equal thickness and processed in an automated tissue processor (TP1020, Leica, Nußloch, Germany), and embedded in paraffin (Microm EC350, Richard-Allan Scientific, Kalamazoo, MI, USA) (Beckstead, 1994; Ismail et al., 2003). Five $\mu \mathrm{m}$ thick sections were mounted on Superfrost Plus glass slides for histology or immunostaining.

\section{MORPHOMETRY AND HISTOLOGY}

Morphometric measurements were performed blindly on tissue sections 4 days and 4 weeks post-MI. Four H\&E-stained (hematoxylin and eosin) section images of each heart were taken at 20x magnification using a DP70 digital camera. Left ventricular (LV) cross-sectional area was measured using Scion imaging software (Scion Corporation, Frederick, MD, USA). LV anterior wall and septal thickness were measured using the average of 4 measurements in each of the 4 sections. The area of infarction was measured 4 days post-MI and taken as an average of total necrotic and granulation tissue areas. Infarct size (IS) was measured and reported as a percentage of the left ventricle.

Myocyte cross-sectional area (MCSA) was measured in 3-8 cardiomyocytes with centrally located nuclei in each of 6 images $(600 \mathrm{x})$ in the endocardium and epicardium ( $n=3-5$ /group) using Scion imaging software (Scion Corporation, Frederick, MD, USA).

Interstitial fibrosis was measured using a Picrosirus Red/Fast Green staining protocol. Briefly, samples were de-paraffinized and rehydrated, immersed in a $0.1 \%$ picrosirius red/fast green solution (Sigma-Aldrich, 365548, F7258, P6744) for 30 minutes, cleared, and coverslipped (Virag et al., 2007). Tissue collagen was determined by taking 4 images at $400 \times$ in 2 sections per heart and using Adobe Photoshop CS4 (Adobe Systems, Mountview, CA) to count the number of red and green pixels. The percent collagen was calculated as the number of red pixels/red + green pixels $\times$ $100 \%$. The numbers were averaged for each animal and the data presented are the average of 3-5 animals per group.

\section{IMMUNOSTAINING}

Tissue sections were deparaffinized in xylene and endogenous peroxidases quenched with $3 \% \mathrm{H}_{2} \mathrm{O}_{2}$ in methanol. Slides were rinsed in saline and incubated with antibodies to CD45 (BD Biosciences; \#550539) for leukocyte infiltration, Ly6G (BD Biosciences, \#550291) for neutrophil infiltration, or CD31 (BD Biosciences, \#553371) for capillary density. For EphA6-R staining, anti-EphA6-R (SantaCruz \#25740) was used. Slides were incubated with appropriate biotinylated secondary antibodies and then with Avidin Biotin Complex (Vector Labs PK-6100). The reaction product was visualized with DAB (Vector, SK-4100), counterstained with methyl green, dehydrated in xylene, and slides were coverslipped.

\section{qRT-PCR}

Whole left ventricles of uninjured (baseline) hearts and hearts 4 days post-MI from WT and EphA2-R-M mice were homogenized using Trizol for RNA isolation. Purification was performed using the Qiagen RNeasy kit. cDNA was made for each sample using a high capacity cDNA kit. Real-time PCR (qRT-PCR) was performed using an Applied Biosystems thermocycler. TaqMan primers were obtained from Applied Biosciences (ephrinA1: Mm00438660_m1, EphA1: Mm00445804_m1, EphA2: Mm00438726_m1, EphA3: Mm00580743_m1, EphA4: Mm00433056_m1, EphA5: Mm00433074_m1, EphA6: Mm00433094_m1, EphA7: Mm00833876_m1, GAPDH: Mm99999915_g1). All samples were run in triplicate and a reaction mixture of $10 \mu \mathrm{l}$ (100 ng RNA) was amplified using recommended conditions. Gene expression was normalized to glyceraldehyde-3-phosphate dehydrogenase (GAPDH) expression. Fluorescence data were analyzed using the $\Delta \Delta \mathrm{Ct}$ method. 


\section{WESTERN BLOTTING}

Whole left ventricles of baseline hearts and hearts 4 days postMI from WT and EphA2-R-M mice were homogenized in a lysis buffer containing $50 \mathrm{mM}$ Hepes, $10 \mathrm{mM}$ EDTA, $100 \mathrm{mM}$ $\mathrm{NaF}, 50 \mathrm{mM}$ sodium pyrophosphate, $1 \%$ protease, and $1 \%$ phosphatase inhibitors. The Bradford Assay was used to quantify the amount of protein. Western blotting was performed on a $4-12 \%$ gradient Bis-Tris gel (BioRad) in $1 \times$ Mops running buffer.

Fifty micrograms of sample was loaded per well. The gel was run for $1 \mathrm{~h}$ at $155 \mathrm{~V}$, and transferred onto pure nitrocellulose membranes (BioRad). Antibodies: GAPDH (Millipore, \#MAB374), p-Akt (Cell Signaling, \#4060), Akt (Cell Signaling, \#4691), matrix metalloproteinase-2 (MMP-2; R\&D Systems, \#AF1488), and NF-кBp65 (Santa Cruz, \#sc-372) followed by appropriate secondary antibodies. All blots were detected with Amersham ECL Advance (GE Healthcare) and imaged on a Typhoon Imager. Densitometry was performed using Image J software (v1.42, NIH, Bethesda, MD) and the intensity of each protein was normalized to GAPDH.

\section{ECHOCARDIOGRAPHY}

Echocardiography was performed on conscious uninjured and infarcted mice at 4 weeks post-MI mice after they had been acclimated in 2-3 sessions within 3 days prior to data acquisition (Yang et al., 1999). A VisualSonics Vevo 2100 diagnostic ultrasound, using M-mode, was used to obtain LV dimensions in diastole and systole. End-diastolic measurements (IVSd, LVPWd, and LVIDd) were obtained at the point of maximal LV diastolic dimension. End-systolic dimensions (IVSs, LVPWs, and LVIDs) were measured at the time of most anterior systolic excursion of the LVPW associated with minimal chamber dimension. Average measurements were calculated using the leading-edge technique of 3- to 5-consecutive sinus beats. Ejection fraction (EF) was calculated from LV dimensions above using the following formula: $\left(\right.$ LVIDd $^{3}-$ LVIDs $\left.^{3}\right) /$ LVIDd $^{3} \times 100 \%$.

\section{STATISTICS}

ANOVA (analysis of variance) with Student-Newman Keuls multiple comparison post-hoc analysis illustrated which groups were statistically significantly different, with significance of at least $p<0.05$.

\section{RESULTS}

\section{BASELINE CHARACTERISTICS}

WT $(n=6)$ mice and EphA2-R-M $(n=7)$ mice weighed $30.22 \pm 1.0 \mathrm{~g}$ and $22.39 \mathrm{~g} \pm 0.5$, respectively $(p<0.001)$. The LV area of EphA2-R-M hearts was $40 \%$ smaller than WT hearts $(p<0.05)$.

LV parameters, $L V$ internal diameter $\left(\mathrm{LV}_{\mathrm{ID}}\right)$, and $\mathrm{LV}$ average wall thickness (AWT) were recorded at baseline (Figure 1). $\mathrm{LV}_{\text {ID was }} 1.49 \pm 0.09 \mathrm{~mm}$ and $1.44 \pm 0.08 \mathrm{~mm}$ in WT $(n=6)$ and EphA2-R-M $(n=7)$ hearts, respectively $(p=0.69)$. LV AWT in WT $(n=6)$ and EphA2-R-M $(n=7)$ hearts was $1.83 \pm 0.06$ and $1.57 \pm 0.05 \mathrm{~mm}$, respectively $(p<0.01)$. No differences in MCSA were observed (data not shown).

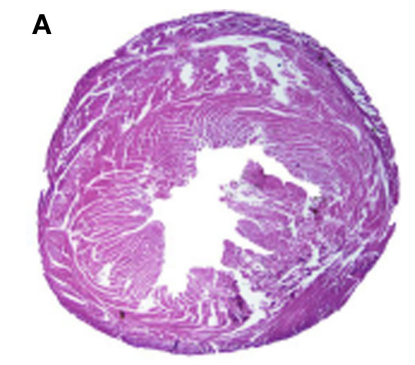

B

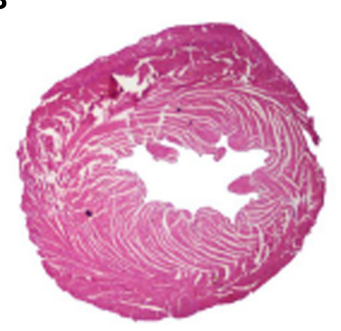

C

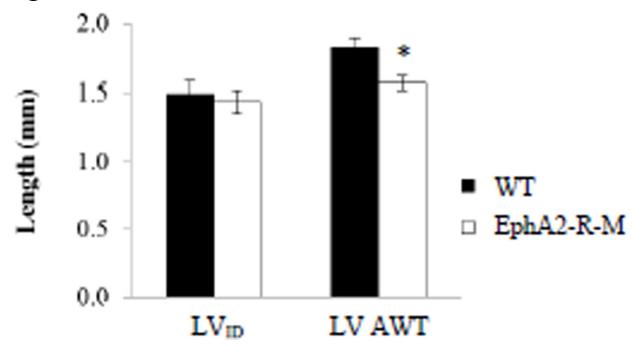

FIGURE 1 | Representative H\&E Stains and Morphometry of Baseline WT and EphA2-R-M Hearts. WT (A) and EphA2-R-M (B) hearts from baseline mice were stained with H\&E (20x). The LV cross-sectional area of the EphA2-R-M hearts was $40 \%$ smaller than WT hearts $(p<0.05)$. At baseline, $\mathrm{LV}_{\mathrm{ID}}$ was $1.49 \pm 0.09 \mathrm{~mm}$ and $1.44 \pm 0.08 \mathrm{~mm}$ in B6 WT $(n=6)$ and EphA2-R-M $(n=7)$ mice, respectively. AWT was $14 \%$ less in EphA2-R-M $(n=7)$ hearts compared with WT $(n=6)$ hearts $\left({ }^{*} p<0.01\right)$ (C) AWT, average wall thickness; $L V$, left ventricle; $L V_{I D}$, Left ventricular internal diameter.

\section{ROLE OF EphA2-R IN ACUTE INJURY Infarct size}

IS was calculated 4 days after infarction in WT $(n=12)$ and EphA2-R-M $(n=9)$ hearts (Figure 2). IS was $37.54 \pm 4.31 \%$ and $49.46 \pm 3.29 \%$ in WT and EphA2-R-M hearts, respectively $(p<0.05)$. Area of necrosis was increased by $113 \%$ in EphA2R-M hearts compared with WT $(p<0.05)$. $\mathrm{LV}_{\text {ID }}$ was increased by $59 \%$ and $53 \%$ in EphA2-R-M $(n=12)$ and WT $(n=10)$ hearts (NS), respectively. No difference in LV AWT was observed in EphA2-R-M $(1.34 \pm 0.05 \mathrm{~mm})$ hearts compared with WT $(1.31 \pm 0.06 \mathrm{~mm})$. There were no differences in MCSA (data not shown).

\section{Ly6-G+ neutrophil density}

Neutrophil infiltration was increased in baseline EphA2-R-M mice and this difference increased by $46 \%$ after MI (Figure 3 ). Ly6-G ${ }^{+}$staining of neutrophil density in WT $(n=6)$ hearts was $0.21 \pm 0.04$ cells $/ 0.1 \mathrm{~mm}^{2}$ and $3.48 \pm 0.29$ cells $/ 0.1 \mathrm{~mm}^{2}$ in EphA2-R-M $(n=3)$ hearts $(p<0.01)$. Ly6-G ${ }^{+}$staining 4 days post-MI in WT $(n=10)$ hearts was $46.96 \pm 4.39$ cells $/ 0.1 \mathrm{~mm}^{2}$ compared with $68.56 \pm 8.59$ cells $/ 0.1 \mathrm{~mm}^{2}$ in EphA2-R-M $(n=9)$ hearts $(p<0.05)$.

\section{$\mathrm{CD}^{+}{ }^{+}$leukocyte density}

Leukocyte density in control animals was no different between WT and EphA2-R-M hearts (Figure 4). CD45 ${ }^{+}$staining of leukocyte infiltration in WT $(n=3)$ mice was $7.63 \pm 1.81$ leukocyte $/ \mu \mathrm{m}^{2}$ compared with $12.54 \pm 2.41$ leukocyte $/ \mu \mathrm{m}^{2}$ in 
A

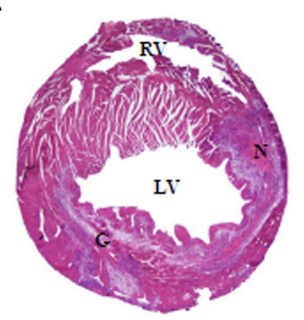

C

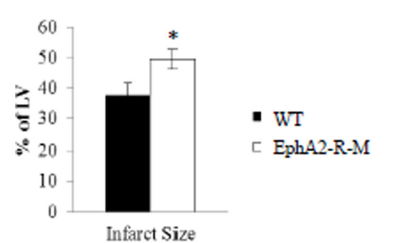

E

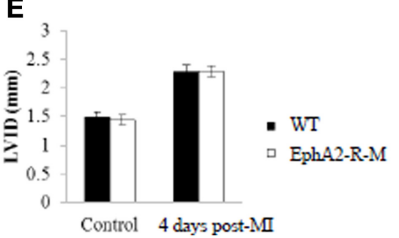

FIGURE 2 | Representative H\&E Stains and Morphometry of WT and EphA2-R-M Hearts 4 Days Post-MI. H\&E Stain of WT (A) and EphA2-R-M (B) hearts 4-days post-MI. Infarct Size (C) was increased by $31.7 \%$ in EphA2-R-M hearts $\left({ }^{*} p<0.05\right)$ and Area of Necrosis (D) was increased by $113 \%$ compared with WT hearts $(* p<0.05)$. LV $V_{I D}$ in EphA2-R-M hearts was increased by $59 \%$ after infarction and increased by $53 \%$ in WT hearts (E) $G$, Granulation Tissue; $L V$, left ventricle; $L V_{I D}$, Left ventricular internal diameter; N, Necrosis; RV, Right Ventricle.

EphA2-RM $(n=10)$ hearts $(p=0.13)$. Leukocyte infiltration was increased by $84 \%$ in EphA2-R-M hearts after MI compared with WT hearts. $\mathrm{CD}^{+} 5^{+}$staining 4 days post-MI in WT $(n=7)$ and EphA2-R-M $(n=8)$ hearts was $55.97 \pm 5.55$ leukocyte $/ \mu \mathrm{m}^{2}$ and $102.76 \pm 5.94$ leukocyte/ $\mu \mathrm{m}^{2}$, respectively $(p<0.001)$.

\section{Western blotting}

A 2.7-fold increase in NF- $\kappa$ B expression was observed in WT $(n=3 ; p<0.01)$ hearts 4 days post-MI compared with no change in EphA2-R-M $(n=3)$ hearts (Figure 5A). Both proand active-MMP-2 were increased 6- and 3-fold in WT mice 4 days post-MI, respectively $(p<0.05)$. No changes were observed in MMP-2 expression in EphA2-R-M hearts 4 days post-MI (Figure 5B). No differences in baseline expression of Akt or p-Akt were observed between EphA2-R-M and WT mice. EphA2-R-M hearts $(n=3)$ had $66.7 \%$ less expression of total Akt protein and $59 \%$ less p-Akt protein than WT $(n=3)$ hearts 4 days post-MI (Figure 5C).

\section{qRT-PCR}

EphrinAl and EphA-R gene expression was quantified using qRTPCR mRNA levels for baseline WT mice $(n=3)$ and EphA2-R-M mice $(n=3)$ (Figure 6C). Although ephrinA1 expression was not different between WT and EphA2-R-M mice at baseline, EphA6

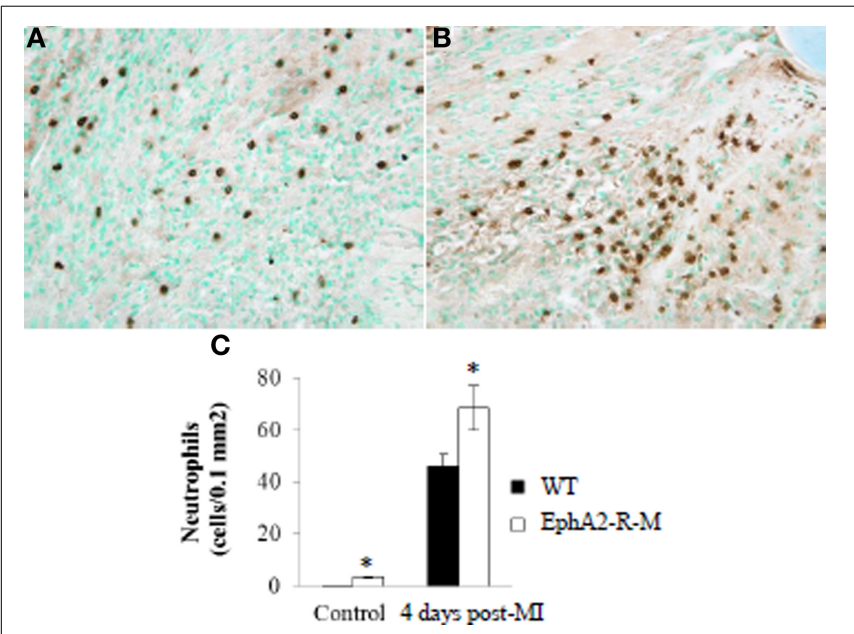

FIGURE 3 | Neutrophil Infiltration in WT and EphA2-R-M Hearts at Baseline and 4 Days Post-MI. Ly6-G+ neutrophil infiltration 4 days Post-MI at 40x magnification in WT (A) and EphA2-R-M (B) hearts. Neutrophil infiltration was increased by $46 \%$ in EphA2-R-M hearts $\left(\mathbf{C}_{;}{ }^{*} p<0.05\right)$.

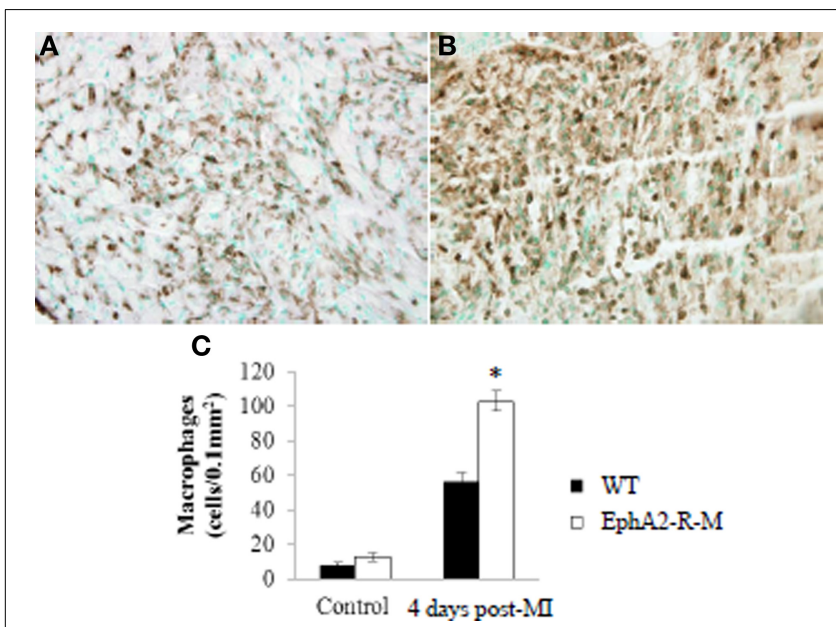

FIGURE 4 | CD45+ Leukocyte Density in WT and EphA2-R-M Hearts at Baseline and 4 Days Post-MI. Leukocyte density staining 4 days post-MI at 40x magnification in WT (A) and EphA2-R-M (B) hearts. Leukocyte infiltration was increased by $84 \%$ in EphA2-R-M hearts after $\mathrm{Ml}$ compared with WT hearts $(\mathbf{C} ; * p<0.001)$.

gene expression was 2.5-fold greater in EphA-R-M (Figure 6B) mice compared with WT (Figure 6A) mice $(p<0.05)$. Of note, EphA3 gene expression was 2.06-fold greater in EphA2-R-M mice compared with WT; however, this difference was not statistically significantly $(p=0.06)$.

EphrinA1 and EphA-R gene expression also was quantified in WT $(n=3)$ and EphA2-R-M $(n=3)$ hearts 4 days postMI (Figure 6F). The expression of ephrinA1 was decreased $42 \%$ in EphA2-R-M hearts compared with WT mice $(p<$ 0.01). EphA6-R expression was 9.8-fold greater in EphA2-R-M (Figure 6E) mice compared with WT (Figure 6D) mice 4 days post-MI $(p=0.01)$. 


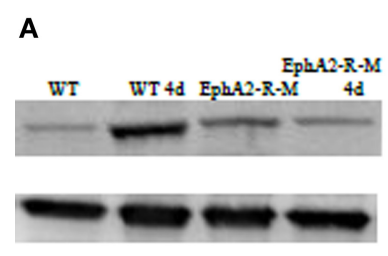

B

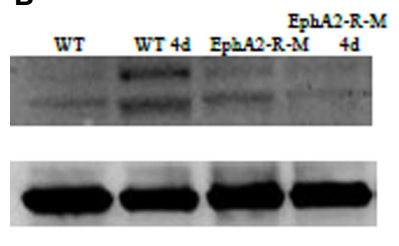

C
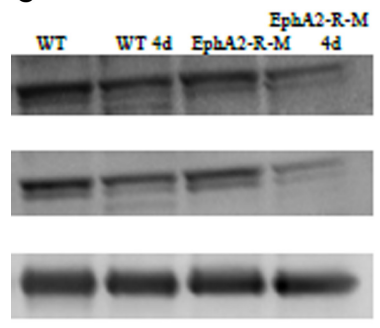

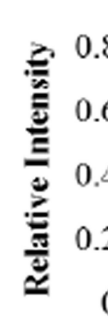

$\mathbf{N F}-\mathbf{k B}$
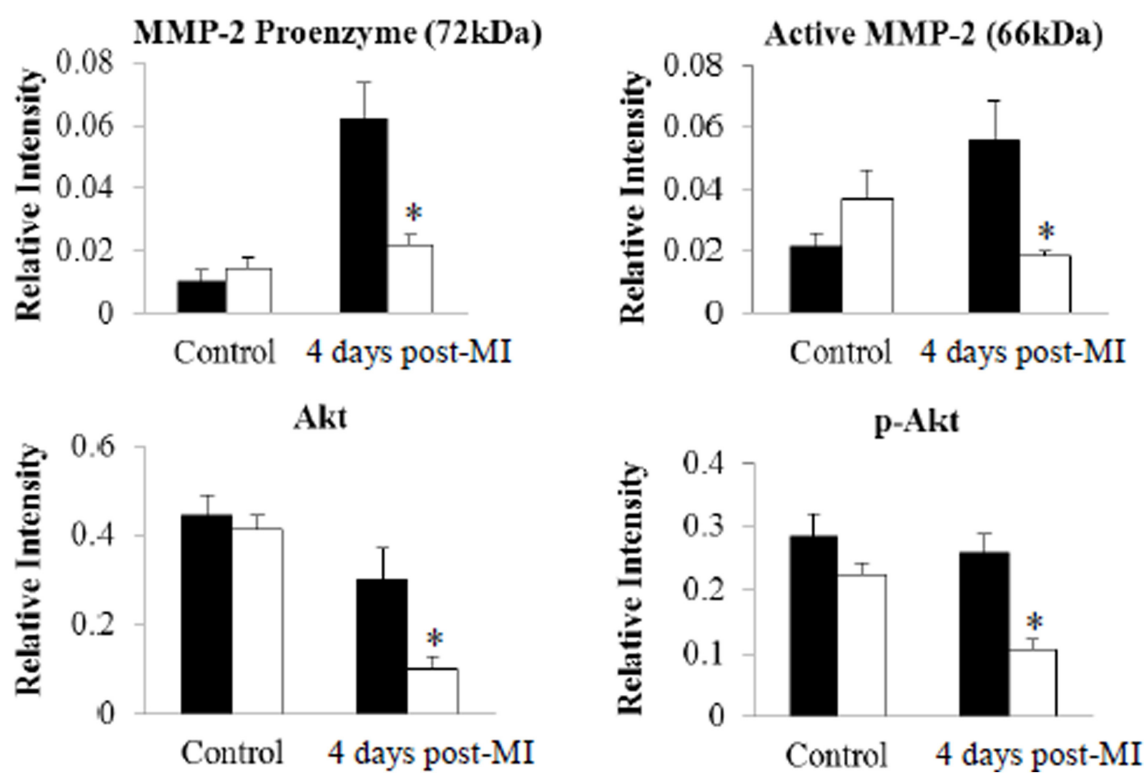

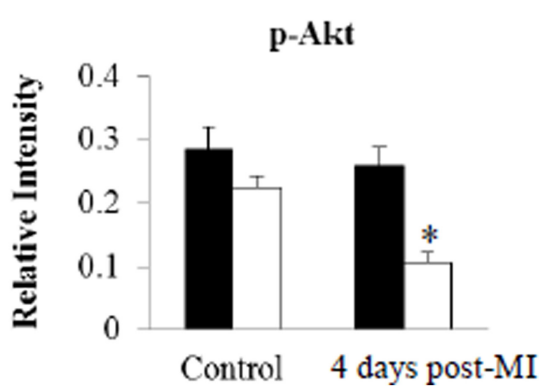

FIGURE 5 | Western Blotting of NF-кB, MMP-2, and Akt in WT, and EphA2-R-M Hearts. NF-кB expression (A) was elevated nearly 3-fold in WT hearts 4 days post-MI compared with baseline $\left({ }^{*} p<0.01\right)$. A similar increase was not observed in EphA2-R-M hearts compared with baseline. Both MMP-2 proenzyme and active form were 6- and 3-fold higher in WT hearts post-MI (B) respectively $\left({ }^{*} p<0.05\right)$. No change in MMP-2 expression was observed in EphA2-R-M hearts. The expression of Akt and p-Akt was significantly decreased in EphA2-R-M hearts post-MI and it was unchanged in WT hearts $\left({ }^{*} p<0.05\right)$ (C).

\section{EphA6-R immunohistochemistry}

Increased expression of EphA6-R in EphA2-R-M mice was noted in mice 4 days post-MI compared with WT mice (Figure 6E). This was supported by the gene expression profile of the EphA6-R observed in EphA2-R-M mice 4 days post-MI (Figure 6F).

\section{ROLE OF THE EPhA2-R IN THE PROGRESSION OF ISCHEMIC CARDIOMYOPATHY \\ Left-ventricular dimensions}

Images of WT and EphA2-R-M hearts 4 weeks post-MI are shown in Figure 7. EphA2-R-M hearts had a $34 \%$ increase in $L V_{\text {ID }}$ compared with WT hearts. WT $(n=6)$ mice observed a $\mathrm{LV}_{\mathrm{ID}}$ of $2.33 \pm 0.21 \mathrm{~mm}$ and EphA2-R-M $(n=5)$ mice had a $\mathrm{LV}_{\mathrm{ID}}$ of $3.10 \pm 0.26 \mathrm{~mm}(p<0.05)$. AWT in WT $(n=6)$ and EphA2-R$\mathrm{M}(n=5)$ mice was $1.28 \pm 0.22 \mathrm{~mm}$ and $0.63 \pm 0.24$, respectively $(p<0.05)$. No differences in MCSA were observed (data not shown).

\section{Fibrosis}

Interstitial fibrosis was not different between WT and EphA2-R$\mathrm{M}$ mice at baseline and 4 weeks post-MI (Figure 8). Interstitial fibrosis, presented as a percentage of the LV, in WT $(n=3)$ hearts was $0.31 \pm 0.22 \%$ compared with $0.10 \pm 0.02 \%$ in EphA2-R$\mathrm{M}(n=5)$ hearts $(p=0.43)$. There was a $26 \%$ increase $(p<$ $0.01)$ in interstitial fibrosis in EphA2-R-M hearts $(2.34 \pm 0.08 \%)$ compared with WT $(1.74 \pm 0.18 \%)$.

\section{Capillary density}

Endocardial capillary density in baseline hearts was 31\% less in EphA2-R-M $(n=5)$ hearts compared with WT $(n=6)$ (WT: $170 \pm 20$ vessels per $400 \mathrm{x}$ high power field; EphA2-R -M: $117 \pm$ 7 vessels per $400 \mathrm{x}$ high power field; $p=0.04$ ) and epicardial capillary density was decreased $24 \%$ in EphA2-R-M mice compared with WT (WT: $134 \pm 15$ vessels per 400x high power field; EphA2R-M: $101 \pm 12$ vessels per $400 \mathrm{x}$ high power field; $p<0.05$ ). Endocardial capillary density 4 days post-MI was reduced by $63 \%$ in EphA2-R-M hearts (Figure 9A). WT mice $(n=4)$ and EphA2-R-M $(n=6)$ endocardial capillary density was $31 \pm 4$ vessels per 400x high power field and $12 \pm 1$ vessels per 400x high power field, respectively ( $p=0.02$ ). In the epicardium 4 days post-MI, there were $27 \pm 2$ vessels per $400 \mathrm{x}$ high power field in WT mice $(n=4)$ and $9 \pm 7$ vessels per $400 x$ high power field in EphA2-R-M mice $(n=6)(p<0.01)$ (Figure 9A). 


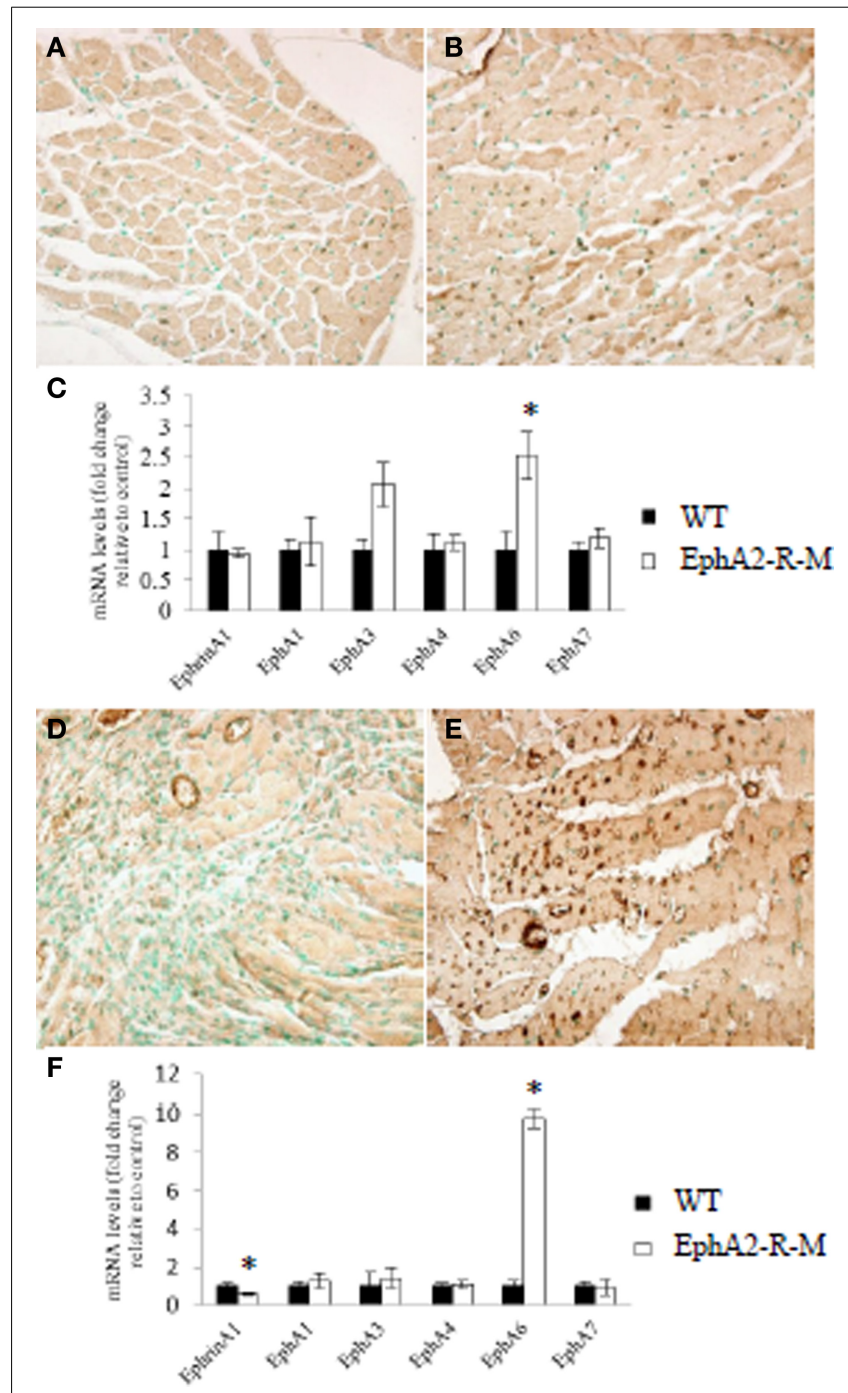

FIGURE 6 | EphA6-R Staining and EphA-R Gene Expression Profiles in WT and EphA2-R-M Hearts at Baseline and 4 Days Post-MI.

Representative immunohistochemical staining at 40x of EphA6-R in WT (A) and EphA2-R-M (B) baseline hearts and 4 days post-MI (WT: D; EphA2-R-M: E). EphrinA1 ligand and EphA-R gene expression in WT and EphA2-R-M hearts was performed in hearts at baseline (C) and 4 days post-MI (F). In baseline hearts, EphA6-R gene expression was 2.5-fold greater in EphA2-R-M hearts ( $\left.{ }^{*} p<0.05\right)$ (C). EphrinA1 ligand expression was decreased $42 \%(* p<0.01)$ and EphA6-R expression was increased 9.8-fold in EphA2-R-M hearts ( $\left.{ }^{*} p<0.01\right)$ (F).

Four weeks post-MI, capillary density in the endocardium of EphA2-R-M hearts was decreased by 53\% compared with WT hearts (Figure 9B). Endocardial capillary density 4 weeks post$\mathrm{MI}$ in WT mice $(n=5)$ and EphA2-R-M mice $(n=3)$ was $32 \pm 2$ vessels per $400 \mathrm{x}$ high power field and $15 \pm 1$ vessels per $400 \mathrm{x}$ high power field, respectively $(p<0.01)$. In the epicardium, capillary density in EphA2-R-M hearts was reduced by $47 \%$ compared with WT (Figure 9B). WT mice $(n=5)$ had $23 \pm$ 1 vessels per $400 \mathrm{x}$ high power field and EphA2-R-M mice $(n=3)$ had a capillary density of $13 \pm 1$ vessels per $400 x$ high power field $(p<0.01)$.

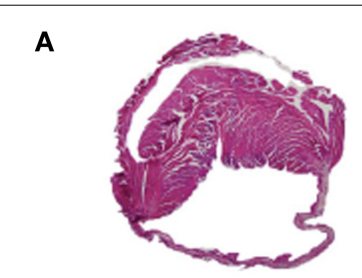

B
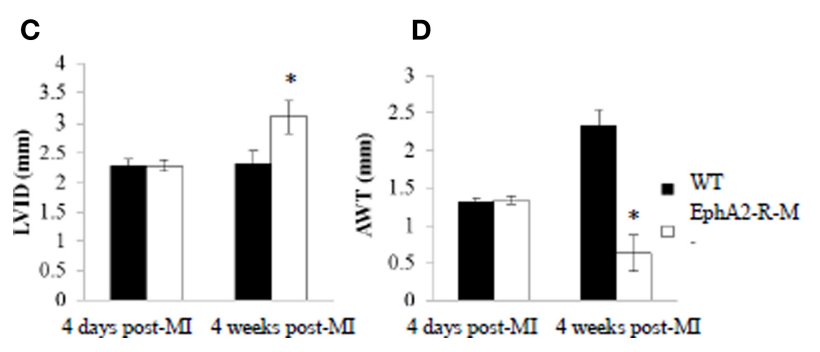

FIGURE 7 | H\&E Stain of WT and EphA2-R Hearts 4 Weeks Post-MI. WT (A) and EphA2-R-M (B) hearts 4 weeks post-MI were stained with $H \& E(20 x) . L_{I D}$ increased by $35 \%(* p<0.05)$ in EphA2-R-M from 4 days to 4 weeks post-MI (C). In WT hearts, LVID was relatively unchanged. AWT decreased by $53 \%\left({ }^{*} p<0.05\right)$ in EphA2-R-M at 4 weeks compared to 4 days while AWT was increased by $78 \%\left({ }^{*} p<0.05\right)$ in WT 4 weeks post-MI (D). LV ID, Left ventricular internal diameter; AWT, average wall thickness.

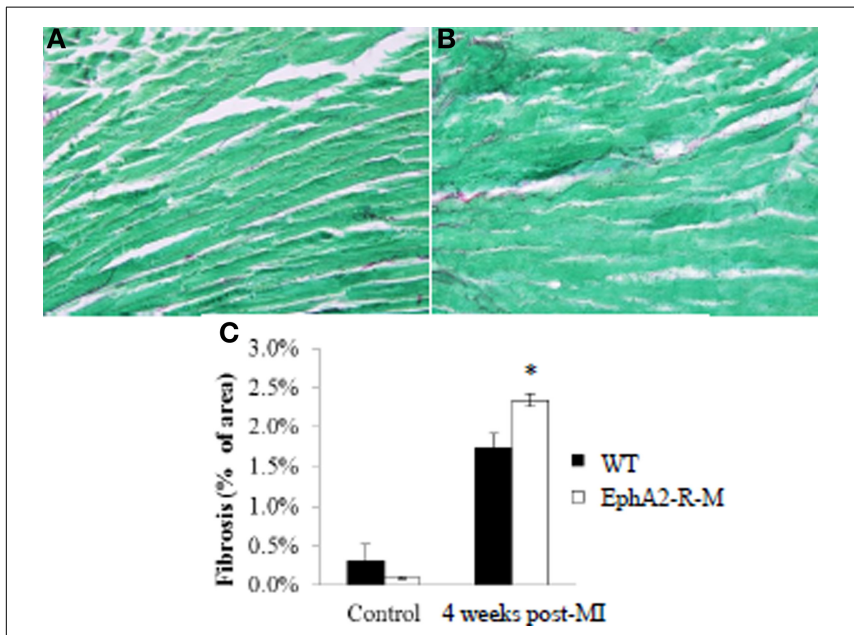

FIGURE 8 | Interstitial Fibrosis in WT and EphA2-R-M Mice 4 Weeks Post-MI. WT (A) and EphA2-R-M (B) images depicting interstitial fibrosis 4 weeks after infarction (400x). EphA2-R-M hearts had 26\% (* $p<0.01)$ more fibrosis present 4 weeks post-MI compared with WT hearts (C).

\section{Cardiac function}

No differences in blood pressure or heart rate in conscious mice between WT and EphA2-R-M mice was observed (data not shown). Cardiac function in EphA2-R-M mice was significantly poorer than WT mice at baseline and 4 weeks post-MI (Figure 10). Baseline ejection fraction in WT $(n=11)$ mice was $94.85 \pm 0.85 \%$ compared with $78.99 \pm 1.51 \%$ in EphA2-R-M $(n=22)$ mice $(p<0.001)$. Ejection fraction 4 weeks post-MI in WT $(n=5)$ and EphA2-R-M $(n=12)$ mice was $64.91 \pm 3.70 \%$ 


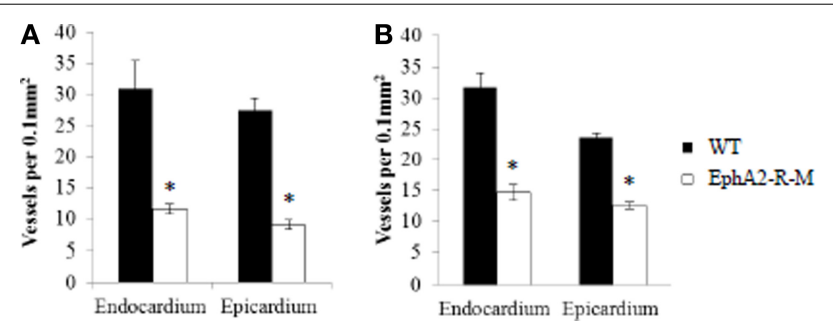

FIGURE 9 | Capillary Density of WT and EphA2-R-M Hearts 4 Days and 4 Weeks Post-MI. Both endocardial and epicardial capillary density were significantly reduced in EphA2-R-M hearts compared with WT 4 days (A) and 4 weeks (B) post-MI $(* p<0.05)$.

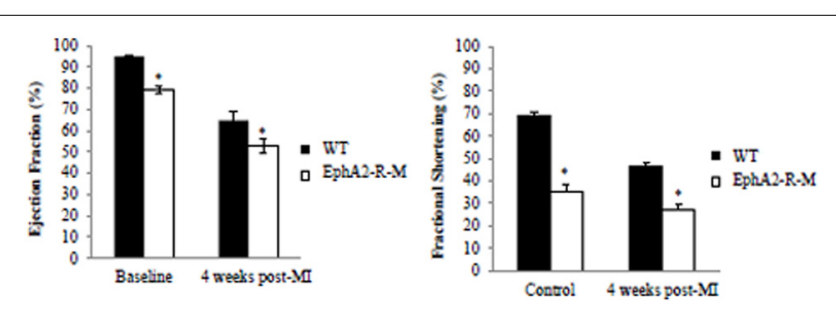

FIGURE 10 | WT and EphA2-R-M Cardiac Function at Baseline and 4 Weeks Post-MI. Baseline EphA2-R-M mice exhibited cardiac dysfunction compared with baseline WT mice and ejection fraction and fractional shortening were depressed in both strains at 4 weeks post-MI with more significant dysfunction observed in EphA2-R-M mice at 4 weeks post-MI (Ejection Fraction: ${ }^{*} p<0.01$; Fractional Shortening: ${ }^{*} p<0.05$ )

and $52.78 \pm 3.59 \%$, respectively $(p=0.04)$. Baseline fractional shortening in WT mice was $68.74 \pm 6.6 \%$ compared with 49.72 $\pm 11.04 \%$ in EphA2-R-M $(p<0.001)$. At 4 weeks post-MI, fractional shortening was $35.40 \pm 6.23 \%$ in WT mice and 26.89 $\pm 7.69 \%$ in EphA2-R-M mice $(p<0.05)$. Chamber dimensions obtained with echocardiographic imaging (data not shown) follow the same trends as those obtained with morphometric analyses.

\section{DISCUSSION}

This is the first report to examine the EphA2-R's role in regulating the cellular events after permanent coronary occlusion and its involvement in the progression of ischemic cardiomyopathy. Specifically, we have demonstrated that the EphA2-R exacerbates the inflammatory response and influences early cardiomyocyte injury that ultimately affects chronic ventricular remodeling.

Currently, the EphA2-R's involvement in regulating acute ischemic injury in the heart is unknown. In a model of ischemic brain injury, Thundyil et al. observed less inflammatory cell infiltrate and reduced apoptosis in EphA2-R-M mice (Thundyil et al., 2013). In contrast, EphA2-R activation protects HL-1 cardiomyocytes from apoptosis and ischemic injury in the kidney (Baldwin et al., 2006; Jehle et al., 2012). These findings provide evidence that the regulation of acute ischemia by the EphA2-R is a tissueand cell-specific phenomenon.

EphrinA reverse signaling has been implicated in regulation of Akt phosphorylation and inhibition of apoptosis (Holen et al., 2008). Additionally, our lab has shown that the upregulation and phosphorylation of Akt protein is associated with improved cardiomyocyte survival in ephrinA1-Fc treated hearts (Brantley-Sieders et al., 2005; Dries et al., 2011). In the current study, we observed decreased expression of Akt and p-Akt in EphA2-R-M hearts 4 days post-MI compared with WT, implicating the EphA2-R's role in modulating cardioprotective Akt signaling.

Necrotic cardiomyocytes trigger an inflammatory response, involving the transmigration of neutrophils and leukocytes which clear debris and release cytokines to promote granulation tissue formation (Frangogiannis et al., 2002; Virag and Murry, 2003; Frangogiannis, 2008, 2012). Neutrophil and macrophage infiltration were significantly increased in EphA2-R-M mice 4 days post-MI while NF- $\kappa \mathrm{B}$ expression was decreased. This paradoxical finding suggests that EphA2-R-M cardiomyocytes have lost their ability to regulate inflammation after infarction. Additionally, the increased residual necrosis and decreased MMP-2 synthesis and activation in EphA2-R-M hearts potentially is explained by defective inflammatory cell function resulting from deficient NF- $\kappa \mathrm{B}$ expression, leading to poorer remodeling and dysfunction. The EphA2-R is a known regulator of inflammation in endothelial cells (Funk et al., 2012; Funk and Orr, 2013) and infiltration of leukocytes occurs in lung tissue deficient of this receptor (Okazaki et al., 2009). Therefore, our work provides evidence that the EphA2-R modulates inflammation in the ischemic myocardium and that defective regulation leads to an elevated inflammatory response. Further studies are needed to examine the cell-specific molecular consequences of NF- $\kappa \mathrm{B}$ deficiency in EphA2-R-M mice.

Macrophages persist in the infarcted area after MI and release chemokines, such as MCP-1 and IL-1 $\beta$, which recruit fibroblasts to deposit collagen to form fibrotic scar tissue. This ultimately leads to progressive ventricular dilation and dysfunction (Nian et al., 2004; Frangogiannis and Entman, 2005). Increased macrophage infiltration and subsequent collagen deposition were observed in EphA2-R-M mice. The resultant enhanced ventricular wall thinning explains the poorer functional capacity of EphA2-R-M mice. Also, a 6-fold change in MMP-2 pro-enzyme expression and a 3 -fold increase in the active form were observed in WT mice 4 days post-MI. However, in EphA2-R-M mice, no significant changes in either form of MMP-2 were observed at baseline or 4 days post-MI. The EphA2-R mediates the acute inflammatory response post-MI and this ultimately influences chronic ventricular remodeling leading to ischemic cardiomyopathy. Cell-specific expression of EphA2-R in the various cells involved in matrix turnover will give further insight into this mechanism.

The EphA2-R is an established angiogenic factor that contributes to vascular development and post-natal angiogenesis. In isolated human brain endothelial cells, inhibition of the EphA2-R with siRNA has been shown to reduce angiogenesis (Zhou et al., 2011). Overexpression of the EphA2-R is associated with increased angiogenesis in ovarian cancer cells and tumorigenesis is impaired in EphA2-R deficient mice (BrantleySieders et al., 2005; Merritt et al., 2011). While angiogenesis in oncogenic pathologies is undesirable, similar mechanisms exist 
as pro-angiogenic stimuli in ischemic tissue which may present a clinical dilemma requiring careful pre-treatment evaluation. The pro-angiogenic properties of the EphA2-R in the heart and in response to ischemia have not been previously described. The capillary density in the endocardium and epicardium was significantly reduced in baseline EphA2-R-M mice and this difference persisted after infarction. This finding supports the role of EphA2-R in vascular development and post-MI neo-angiogenesis in the murine heart.

To our knowledge, the functional capacity of mice deficient of the EphA2-R has not been reported. At baseline, ejection fraction of EphA2-R-M mice was slightly but significantly reduced compared with WT animals, suggesting a developmental role of the EphA2-R in the functional capacity of the heart. The EphA2-R has been implicated in spinal neuralation (AbdulAziz et al., 2009). However, no literature exists on its role in cardiac development or in cardiac physiology. Decreased capillary density potentially explains the poorer function of the EphA2-R-M mice. This would result in a limited ability of the myocardium to respond to alterations in perfusion or oxygen concentration. Although we have not performed functional analyses of the vessels or determined whether this deficiency alters perfusion, it is possible that the inadequate revascularization we observed post-MI explains our findings in the EphA2-R-M myocardium.

Our previous work has reported that the expression profiles of the ephrinA1 ligand and the EphA-Rs (A1-4, 6, and 7) change in the heart after MI and with treatment of exogenous ephrinA1Fc ligand (Dries et al., 2011). In our prior study, the EphA6-R was downregulated and the EphA2-R was reciprocally upregulated in response to MI and with ephrinA1-Fc treatment. The current study supports the reciprocal expression profile of the EphA2-R and the EphA6-R as EphA6-R expression was increased in EphA2-R-M mice. Potentially, significant cross-talk between these receptors exist to alter activation or inhibition of each receptor such that EphA6 upregulation in the EphA2-R-M offsets the deleterious effects of the absence of EphA2-R. This is supported by the fact that EphA and EphB receptors have been shown to form heterotypic clusters upon ligand binding and activation or inhibition of the signaling cascades depends on these receptors' specific interactions (Janes et al., 2011). The EphA2-R most likely mediates cardioprotection and interacts with the EphA6-R to decrease potential negative sequelae mediated through this receptor. Further studies to characterize the cell-specific expression profiles of the EphA2-R and EphA6-R are needed to elucidate their interaction and role in post-MI myocardium.

Expression of the protective ephrinA1 ligand was significantly decreased in EphA2-R-M mice after MI. This is consistent with our previous work (Dries et al., 2011). The reduction in the ephrinA1 ligand gene expression in our current study supports our previous finding that the cardioprotective benefits of endogenous ephrinA1 are lost after MI and worsened in EphA2-R-M mice. Since this study does not explore the potential of ephrinA1 treatment to reduce injury during MI, further studies are warranted to determine if the cardioprotective benefits of this ligand can be reproduced in mice that lack the EphA2-R.
In conclusion, this study has demonstrated the complex role of the EphA2-R in the heart after MI. The EphA2-R is a critical mediator of inflammation and early injury during acute MI which influences the progression of ischemic cardiomyopathy. EphrinA1-EphA signaling represents an exciting new area of exploration in the heart and further studies are needed to determine their role and therapeutic potential to blunt injury in MI.

\section{AUTHOR CONTRIBUTIONS}

Wesley T. O’Neal and William F. Griffin compiled, analyzed, and interpreted all data and drafted the manuscript. Susan D. Kent carried out the mouse breeding, performed the RT-PCR, western blotting, histology and immunohistochemistry, and blood pressure experiments. Filza Faiz, Jonathan Hodges, and Jackson Vuncannon assisted in collecting and compiling data and editing the manuscript. Jitka A. I. Virag designed the study, obtained the funding, surgical procedures, and echocardiography, supervised data collection, analyzed and interpreted data, and edited the manuscript to ensure accuracy and inclusion of important intellectual content. All authors have read the manuscript and have given final approval of this version.

\section{ACKNOWLEDGMENTS}

Jitka Virag is supported by the North Carolina Biotechnology Center, the Department of Physiology, the Department of Comparative Medicine, and the East Carolina Diabetes and Obesity Institute.

\section{REFERENCES}

Abdul-Aziz, N. M., Turmaine, M., Greene, N. D., and Copp, A. J. (2009). EphrinAEphA receptor interactions in mouse spinal neurulation: implications for neural fold fusion. Int. J. Dev. Biol. 53, 559-568. doi: 10.1387/ijdb.082777na

Baldwin, C., Chen, Z. W., Bedirian, A., Yokota, N., Nasr, S. H., Rabb, H., et al. (2006). Upregulation of EphA2 during in vivo and in vitro renal ischemiareperfusion injury: role of Src kinases. Am. J. Physiol. Renal. Physiol. 291, F960-F971. doi: 10.1152/ajprenal.00020.2006

Beauchamp, A., and Debinski, W. (2011). Ephs and ephrins in cancer: ephrinA1 signalling. Semin. Cell Dev. Biol. 23, 109-115. doi: 10.1016/j.semcdb.2011. 10.019

Beckstead, J. H. (1994). A simple technique for preservation of fixation-sensitive antigens in paraffin-embedded tissues. J. Histochem. Cytochem. 42, 1127-1134. doi: $10.1177 / 42.8 .8027531$

Brantley, D. M., Cheng, N., Thompson, E. J., Lin, Q., Brekken, R. A., Thorpe, P. E., et al. (2002). Soluble Eph A receptors inhibit tumor angiogenesis and progression in vivo. Oncogene 21, 7011-7026. doi: 10.1038/sj.onc.1205679

Brantley-Sieders, D. M., Caughron, J., Hicks, D., Pozzi, A., Ruiz, J. C., and Chen, J. (2004a). EphA2 receptor tyrosine kinase regulates endothelial cell migration and vascular assembly through phosphoinositide 3-kinase-mediated Racl GTPase activation. J. Cell Sci. 117(Pt 10), 2037-2049. doi: 10.1242/ jcs.01061

Brantley-Sieders, D. M., Fang, W. B., Hicks, D. J., Zhuang, G., Shyr, Y., and Chen, J. (2005). Impaired tumor microenvironment in EphA2-deficient mice inhibits tumor angiogenesis and metastatic progression. FASEB J. 19, 1884-1886. doi: 10.1096/fj.05-4038fje

Brantley-Sieders, D. M., Fang, W. B., Hwang, Y., Hicks, D., and Chen, J. (2006). Ephrin-A1 facilitates mammary tumor metastasis through an angiogenesisdependent mechanism mediated by EphA receptor and vascular endothelial growth factor in mice. Cancer Res. 66, 10315-10324. doi: 10.1158/00085472.CAN-06-1560

Brantley-Sieders, D., Schmidt, S., Parker, M., and Chen, J. (2004b). Eph receptor tyrosine kinases in tumor and tumor microenvironment. Curr. Pharm. Des. 10, 3431-3442. doi: 10.2174/1381612043383160 
Cheng, N., Brantley, D. M., and Chen, J. (2002). The ephrins and Eph receptors in angiogenesis. Cytokine Growth Factor Rev. 13, 75-85. doi: 10.1016/S13596101(01)00031-4

Coulthard, M. G., Morgan, M., Woodruff, T. M., Arumugam, T. V., Taylor, S. M., Carpenter, T. C., et al. (2012). Eph/Ephrin signaling in injury and inflammation. Am. J. Pathol. 181, 1493-1503. doi: 10.1016/j.ajpath.2012. 06.043

Deroanne, C., Vouret-Craviari, V., Wang, B., and Pouyssegur, J. (2003). EphrinA1 inactivates integrin-mediated vascular smooth muscle cell spreading via the Rac/PAK pathway. J. Cell Sci. 116(Pt 7), 1367-1376. doi: 10.1242/jcs.00308

Dries, J. L., Kent, S. D., and Virag, J. A. (2011). Intramyocardial administration of chimeric ephrinA1-Fc promotes tissue salvage following myocardial infarction in mice. J. Physiol. 589(Pt 7), 1725-1740. doi: 10.1113/jphysiol.2010.202366

Frangogiannis, N. G. (2008). The immune system and cardiac repair. Pharmacol. Res. 58, 88-111. doi: 10.1016/j.phrs.2008.06.007

Frangogiannis, N. G. (2012). Regulation of the inflammatory response in cardiac repair. Circ. Res. 110, 159-173. doi: 10.1161/CIRCRESAHA.111.243162

Frangogiannis, N. G., and Entman, M. L. (2005). Chemokines in myocardial ischemia. Trends Cardiovasc. Med. 15, 163-169. doi: 10.1016/j.tcm.2005.06.005

Frangogiannis, N. G., Smith, C. W., and Entman, M. L. (2002). The inflammatory response in myocardial infarction. Cardiovasc. Res. 53, 31-47. doi: 10.1016/S0008-6363(01)00434-5

French, C. J., Taatjes, D. J., and Sobel, B. E. (2010). Autophagy in myocardium of murine hearts subjected to ischemia followed by reperfusion. Histochem. Cell Biol. 134, 519-526. doi: 10.1007/s00418-010-0748-0

Funk, S. D., and Orr, A. W. (2013). Ephs and ephrins resurface in inflammation, immunity, and atherosclerosis. Pharmacol. Res. 67, 42-52. doi: 10.1016/j.phrs.2012.10.008

Funk, S. D., Yurdagul, A. Jr., Albert, P., Traylor, J. G. Jr., Jin, L., Chen, J., et al. (2012). EphA2 activation promotes the endothelial cell inflammatory response: a potential role in atherosclerosis. Arterioscler. Thromb. Vasc. Biol. 32, 686-695. doi: 10.1161/ATVBAHA.111.242792

Goichberg, P., Bai, Y., D’Amario, D., Ferreira-Martins, J., Fiorini, C., Zheng, H., et al. (2011). The ephrin A1-EphA2 system promotes cardiac stem cell migration after infarction. Circ. Res. 108, 1071-1083. doi: 10.1161/CIRCRESAHA.110.239459

Holen, H. L., Shadidi, M., Narvhus, K., Kjosnes, O., Tierens, A., and Aasheim, H. C. (2008). Signaling through ephrin-A ligand leads to activation of Src-family kinases, Akt phosphorylation, and inhibition of antigen receptor-induced apoptosis. J. Leukoc. Biol. 84, 1183-1191. doi: 10.1189/jlb.1207829

Incerti, M., Tognolini, M., Russo, S., Pala, D., Carmine, G., Hassan-Mohamed, I., et al. (2013). Amino acid conjugates of lithocholic acid as antagonists of the EphA2 receptor. J. Med. Chem. 56, 2936-2947. doi: 10.1021/jm301890k

Ismail, J. A., Poppa, V., Kemper, L. E., Scatena, M., Giachelli, C. M., Coffin, J. D., et al. (2003). Immunohistologic labeling of murine endothelium. Cardiovasc. Pathol. 12, 82-90. doi: 10.1016/S1054-8807(02)00166-7

Ivanov, A. I., and Romanovsky, A. A. (2006). Putative dual role of ephrinEph receptor interactions in inflammation. IUBMB Life 58, 389-394. doi: $10.1080 / 15216540600756004$

Janes, P. W., Griesshaber, B., Atapattu, L., Nievergall, E., Hii, L. L., Mensinga, A., et al. (2011). Eph receptor function is modulated by heterooligomerization of A and B type Eph receptors. J. Cell Biol. 195, 1033-1045. doi: $10.1083 /$ jcb. 201104037

Jehle, J., Staudacher, I., Wiedmann, F., Schweizer, P., Becker, R., Katus, H., et al. (2012). Regulation of apoptosis in HL-1 cardiomyocytes by phosphorylation of the receptor tyrosine kinase EphA2 and protection by lithocholic acid. Br. J. Pharmacol. 167, 1563-1572. doi: 10.1111/j.1476-5381.2012.02117.x

Jugdutt, B. I. (2012). Ischemia/Infarction. Heart Fail. Clin. 8, 43-51. doi: 10.1016/j.hfc.2011.08.006

Kannel, W. B. (2000). Incidence and epidemiology of heart failure. Heart Fail. Rev. 5, 167-173. doi: 10.1023/A:1009884820941

Kullander, K., and Klein, R. (2002). Mechanisms and functions of Eph and ephrin signalling. Nat. Rev. Mol. Cell Biol. 3, 475-486. doi: 10.1038/nrm856

Kung, G., Konstantinidis, K., and Kitsis, R. N. (2011). Programmed necrosis, not apoptosis, in the heart. Circ. Res. 108, 1017-1036. doi: 10.1161/CIRCRESAHA. 110.225730
McCarron, J. K., Stringer, B. W., Day, B. W., and Boyd, A. W. (2010). Ephrin expression and function in cancer. Future Oncol. 6, 165-176. doi: 10.2217/fon. 09.146

Merritt, W. M., Kamat, A. A., Hwang, J. Y., Bottsford-Miller, J., Lu, C., Lin, Y. G., et al. (2011). Clinical and biological impact of EphA2 overexpression and angiogenesis in endometrial cancer. Cancer Biol. Ther. 10, 1306-1314. doi: 10.4161/cbt.10.12.13582

Miao, H., Li, D. Q., Mukherjee, A., Guo, H., Petty, A., Cutter, J., et al. (2009). EphA2 mediates ligand-dependent inhibition and ligand-independent promotion of cell migration and invasion via a reciprocal regulatory loop with Akt. Cancer Cell 16, 9-20. doi: 10.1016/j.ccr.2009.04.009

Nian, M., Lee, P., Khaper, N., and Liu, P. (2004). Inflammatory cytokines and postmyocardial infarction remodeling. Circ. Res. 94, 1543-1553. doi: 10.1161/01.RES.0000130526.20854.fa

Okazaki, T., Ni, A., Baluk, P., Ayeni, O. A., Kearley, J., Coyle, A. J., et al. (2009). Capillary defects and exaggerated inflammatory response in the airways of EphA2-deficient mice. Am. J. Pathol. 174, 2388-2399. doi: 10.2353/ajpath.2009.080949

O’Neal, W. T., Griffin, W. F., Dries-Devlin, J. L., Kent, S. D., Chen, J., Willis, M. S., et al. (2013). Ephrin-Eph signaling as a potential therapeutic target for the treatment of myocardial infarction. Med. Hypotheses 80, 738-744. doi: 10.1016/j.mehy.2013.02.024

Pandey, A., Shao, H., Marks, R. M., Polverini, P. J., and Dixit, V. M. (1995). Role of B61, the ligand for the Eck receptor tyrosine kinase, in TNF-alpha-induced angiogenesis. Science 268, 567-569. doi: 10.1126/science.7536959

Pasquale, E. B. (2008). Eph-ephrin bidirectional signaling in physiology and disease. Cell 133, 38-52. doi: 10.1016/j.cell.2008.03.011

Sakamoto, A., Sugamoto, Y., Tokunaga, Y., Yoshimuta, T., Hayashi, K., Konno, T., et al. (2011). Expression profiling of the ephrin (EFN) and Eph receptor (EPH) family of genes in atherosclerosis-related human cells. J. Int. Med. Res. 39, 522-527. doi: 10.1177/147323001103900220

Thundyil, J., Manzanero, S., Pavlovski, D., Cully, T. R., Lok, K. Z., Widiapradja, A., et al. (2013). Evidence that the EphA2 receptor exacerbates ischemic brain injury. PLoS ONE 8:e53528. doi: 10.1371/journal.pone.0053528

Virag, J. A., Rolle, M. L., Reece, J., Hardouin, S., Feigl, E. O., and Murry, C. E. (2007). Fibroblast growth factor-2 regulates myocardial infarct repair: effects on cell proliferation, scar contraction, and ventricular function. Am. J. Pathol. 171, 1431-1440. doi: 10.2353/ajpath.2007.070003

Virag, J. I., and Murry, C. E. (2003). Myofibroblast and endothelial cell proliferation during murine myocardial infarct repair. Am. J. Pathol. 163, 2433-2440. doi: 10.1016/S0002-9440(10)63598-5

Yang, X. P., Liu, Y. H., Rhaleb, N. E., Kurihara, N., Kim, H. E., and Carretero, O. A. (1999). Echocardiographic assessment of cardiac function in conscious and anesthetized mice. Am. J. Physiol. 277(5 Pt 2), H1967-H1974.

Zhou, N., Zhao, W. D., Liu, D. X., Liang, Y., Fang, W. G., Li, B., et al. (2011). Inactivation of EphA2 promotes tight junction formation and impairs angiogenesis in brain endothelial cells. Microvasc. Res. 82, 113-121. doi: 10.1016/j.mvr.2011.06.005

Conflict of Interest Statement: The authors declare that the research was conducted in the absence of any commercial or financial relationships that could be construed as a potential conflict of interest.

Received: 11 February 2014; accepted: 17 March 2014; published online: 24 April 2014 Citation: O'Neal WT, Griffin WF, Kent SD, Faiz F, Hodges J, Vuncannon J and Virag JAI (2014) Deletion of the EphA2 receptor exacerbates myocardial injury and the progression of ischemic cardiomyopathy. Front. Physiol. 5:132. doi: 10.3389/fphys. 2014.00132

This article was submitted to Integrative Physiology, a section of the journal Frontiers in Physiology.

Copyright (C) 2014 O'Neal, Griffin, Kent, Faiz, Hodges, Vuncannon and Virag. This is an open-access article distributed under the terms of the Creative Commons Attribution License (CC BY). The use, distribution or reproduction in other forums is permitted, provided the original author(s) or licensor are credited and that the original publication in this journal is cited, in accordance with accepted academic practice. No use, distribution or reproduction is permitted which does not comply with these terms. 